DOI: http://dx.doi.org/10.22483/2177-5796.2018v20n2p441-453

\title{
O uso do Dojo na prática pedagógica do ensino de lógica de programação
}

\author{
Fabio Gomes Rocha \\ Ester Fraga Vilas-Bôas Carvalho do Nascimento
}

Resumo: O objetivo deste trabalho foi analisar a contribuição do uso de softwares para o ensino de lógica, adotandose o "Dojo" como prática pedagógica. Como investigação empírica, a pesquisa constitui um estudo de caso, na disciplina de Lógica de Programação do curso Técnico de Informática do Serviço Nacional de Aprendizagem Industrial do Estado de Sergipe. A partir da verificação sobre as dificuldades de aprendizagem dos estudantes, desenvolveu-se à experimentação do uso do Dojo como metodologia para o ensino. As análises foram feitas sob abordagem qualitativa, associando-se os dados obtidos na atividade pedagógica e os referenciais adotados no estudo. Os resultados apontaram a redução do número de faltas, a satisfação do aluno e a elevação da média nas avaliações da disciplina, constatando a viabilidade de adoção da prática experimentada para o sucesso na aprendizagem.

Palavras-chave: Dojo. Educação profissional. Lógica de programação. Práticas pedagógicas.

\section{The use of the Dojo in the pedagogical practice of teaching programming logic}

Abstract: The objective of this work was to analyze the contribution of the use of software for the teaching of logic, adopting the "Dojo" as pedagogical practice. As an empirical investigation, the research is a case study, in the discipline of Programming Logic of the Technical Course of Informatics, on Serviço Nacional de Aprendizagem Industrial of Sergipe, Brazil. Based on the verification of students' learning difficulties, they developed the experimentation of using the "Dojo" as a methodology for teaching. The analyzes were realized under a qualitative approach, combining the data obtained in the pedagogical activity and the references adopted in the study. The results indicated a reduction in the number of absences, student satisfaction and the average increase in the discipline evaluations, confirming the feasibility of adopting the practice for learning success.

Keywords: Dojo. Professional education. Programming logic. Pedagogical practices. 
ROCHA, Fabio Gomes; NASCIMENTO, Ester Fraga Vilas-Bôas Carvalho do. O uso do Dojo na prática pedagógica do ensino de lógica de programação.

\section{Introdução}

A evolução tecnológica ocorrida entre as décadas de 1980 e 1990, com o surgimento do microcomputador e da Internet, deu origem a novas ocupações e impulsionou a de programador de computador. A alta aplicabilidade dos softwares, desde a gestão de usinas nucleares à disponibilização de jogos eletrônicos para celulares (SEBESTA, 2013), proporcionou a expansão da demanda de mão de obra qualificada para esse trabalho. A formação especializada para a programação de computadores surge, assim, como resposta a uma necessidade social (DEWEY, 1965), sendo iniciada nos Estados Unidos em 1962 com cursos superiores (RICE; ROSEN, 1994). No Brasil, os primeiros cursos superiores de Ciências da Computação foram criados em 1969 na Universidade de Campinas (UNICAMP) e Universidade Federal da Bahia (UFBA) (CABRAL et al., 2008).

A ramificação dessa formação em curso de curta duração, então intitulado "Técnico de Nível Superior" foi incentivada no âmbito das políticas governamentais conduzidas pela Comissão de Coordenação das Atividades de Processamento Eletrônico (CAPRE), criada pelo Decreto $\mathrm{n}^{\mathrm{o}}$ 70.370, de 5 de abril de 1972 (BRASIL, 1972a). Entre os objetivos da comissão constava: “[...] adotar e propor medidas visando à racionalização dos investimentos governamentais no setor e à elevação da produtividade na utilização dos equipamentos de processamento de dados instalados e a instalar" (BRASIL, 1972a, p. 1). No ano seguinte ao início de suas atividades, a CAPRE forneceu apoio orçamentário para a implantação do "Curso Superior de Processamento de Dados", com duração entre dois a quatro anos, na Pontifícia Universidade Católica do Rio de Janeiro (PUC-RIO), Universidade Federal de Minas Gerais (UFMG), Universidade Federal da Paraíba (UFPB) e Universidade Federal do Rio Grande do Sul (UFRGS) (CABRAL et al., 2008).

Essas iniciativas, no entanto, pareciam não atender à demanda de recursos humanos para as atividades de programação requeridas pelo mercado. Uma alternativa para a aceleração da formação nesse campo foi construída no âmbito do ensino técnico, em nível médio, com os cursos de Processamento de Dados. Inicialmente, o Parecer no 45, de 12 de janeiro de 1972, que tratou sobre os requisitos mínimos para as habilitações profissionais no ensino médio, indicava a possibilidade das escolas criarem o curso de "Auxiliar de Processamento de Dados". Essa formação foi alocada na categoria "outras habilitações", as quais poderiam atender a demandas 
ROCHA, Fabio Gomes; NASCIMENTO, Ester Fraga Vilas-Bôas Carvalho do. O uso do Dojo na prática pedagógica do ensino de lógica de programação.

locais ou regionais, mas os certificados ou diplomas correspondentes não teriam validade nacional (BRASIL, 1972b).

A capilaridade que a oferta dessa formação poderia assumir em nível estadual surge como obstáculo para o estabelecimento de um marco sobre o início dos referidos cursos no Brasil. No entanto, observa-se a organização coletiva de trabalhadores em processamento de dados a partir de 1977, com a criação da Associação dos Profissionais em Processamento de Dados (APPD) no Rio Grande do Sul (RS). Embora pioneiro nessa ação, o estado só veio a ofertar a formação profissionalizante em Processamento de Dados, em instituição pública, em 1989, na Escola de Técnica de Comércio da UFGRS. Ainda em década anterior, a Escola Técnica Federal de São Paulo, atual Instituto Federal de São Paulo (IFSP), deu início ao curso Técnico em Processamento de Dados (INSTITUTO FEDERAL DE SÃO PAULO, 2017).

Em âmbito internacional, a relevância da formação técnica no campo da computação pode ser observada no reconhecimento como uma das categorias de conhecimento da WorldSkills International, evento mundial e bianual que se realiza há 67 anos, contando com a participação de 52 países membros (WORLDSKILLS, 2010). A coordenação brasileira é realizada pelo Serviço Nacional de Aprendizagem Industrial (SENAI), que a partir 1983 passou a inserir seus alunos nas provas de conhecimento. Em 1993 ocorreu a primeira participação dos estudantes dos cursos técnicos profissionalizantes em informática daquela instituição, obtendo medalha de ouro nos anos de 2001, 2007 e 2009. A partir desse último ano, os estudantes brasileiros obtiveram sucessivamente posição entre três primeiras premiações na competição. A seguir, são expostas imagens dos momentos de provas em computação e da comitiva de alunos brasileiros. 
ROCHA, Fabio Gomes; NASCIMENTO, Ester Fraga Vilas-Bôas Carvalho do. O uso do Dojo na prática pedagógica do ensino de lógica de programação.

Figura 1 - Estudantes de educação profissionalizante em prova de "soluções de softwares", na WorldSkills International de 2004

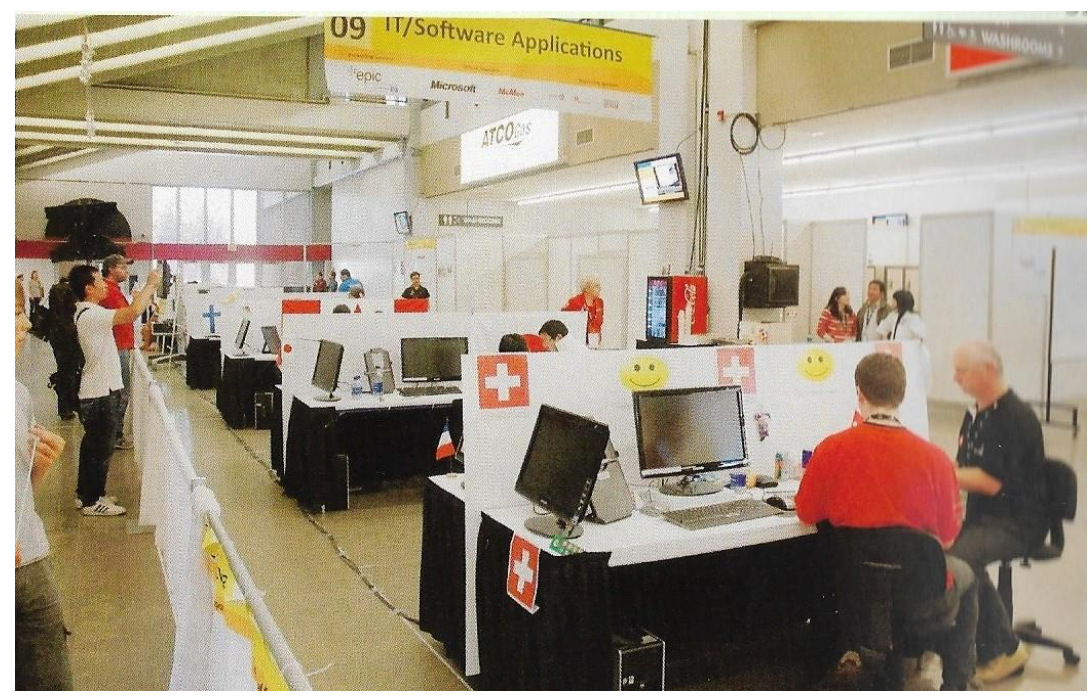

Fonte: WORLDSKILLS. WorldSkills International History. Santo André: Ipsis Gráfica, 2010. p. 18.

Figura 2 - Estudantes brasileiros de educação profissionalizante na WorldSkills International, em 2015

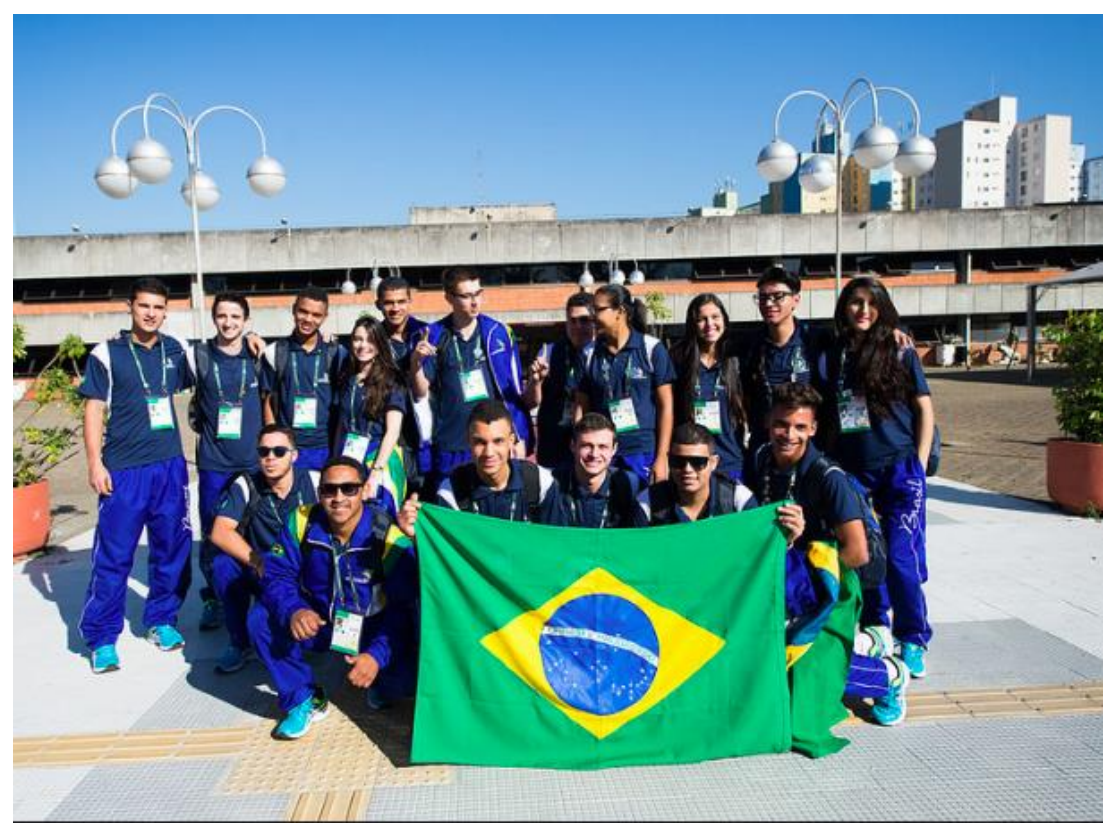

Fonte: WORLDSKILLS. Site oficial de fotografias da WorldSkills. Disponível em: < https://www.flickr.com/photos/worldskills/>. Acesso em: 25 maio 2017. 
ROCHA, Fabio Gomes; NASCIMENTO, Ester Fraga Vilas-Bôas Carvalho do. O uso do Dojo na prática pedagógica do ensino de lógica de programação.

Figura 3 - Estudante brasileiro de educação profissionalizante do SENAI em prova de "soluções de softwares", na WorldSkills International de 2015

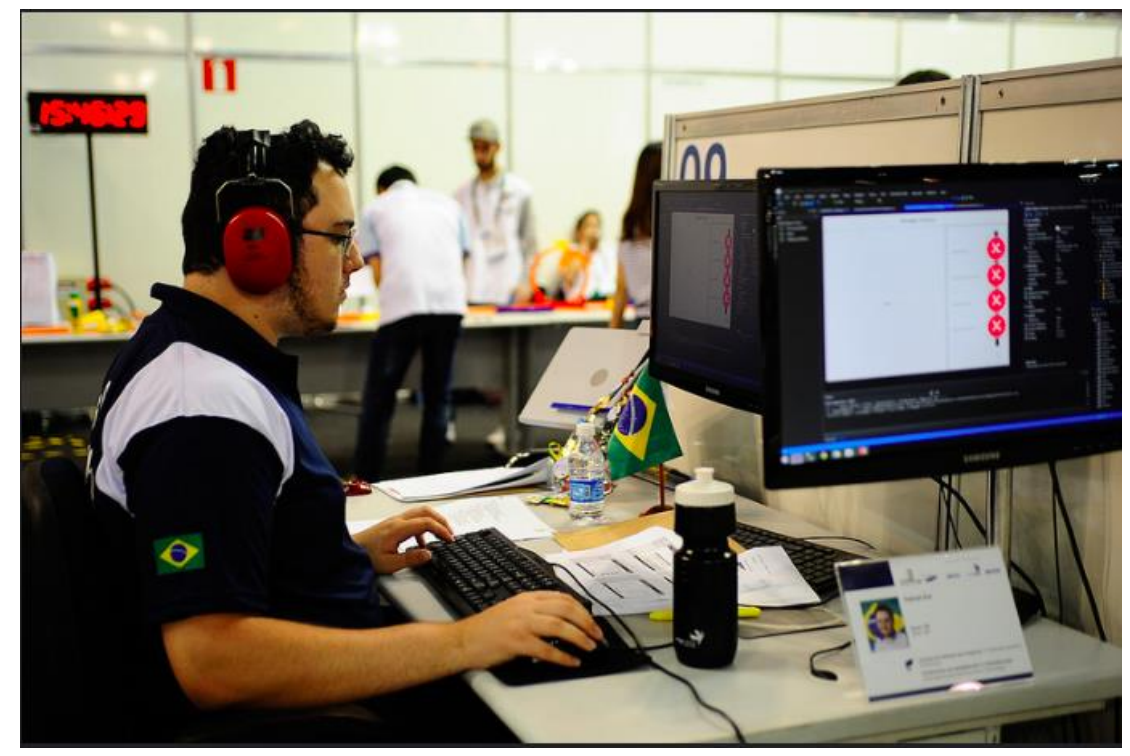

Fonte: WORLDSKILLS. Site oficial de fotografias da WorldSkills. Disponível em: < https://www.flickr.com/photos/worldskills/>. Acesso em: 25 maio 2017.

A expectativa sobre os conhecimentos a serem desenvolvidos para os técnicos em informática está envolta de questões que se relacionam ao pensamento sistematizado, abstração de dados e foco na solução de problemas como um sistema. Esses aspectos são encontrados na disciplina de Lógica de Programação, cujo o foco é estudar "[...] as leis e os critérios de validade que regem o pensamento e a demonstração" (MANZANO; OLIVEIRA, 2009, p. 3). Sendo uma aprendizagem essencial para a programação de computadores e, portanto, para as carreiras relacionadas à informática (PIMENTEL et al., 2003), a disciplina de Lógica de Programação é ministrada na primeira fase do curso, onde os discentes devem aprender a desenvolver o raciocínio lógico para a resolução de problemas.

Devido à sua natureza, relacionada a operações mentais que envolvem percepção e análises apuradas para a elaboração do conhecimento para soluções, a disciplina é uma das principais razões para evasão e reprovação nas primeiras fases dos cursos de Técnico em Informática (SILVEIRA, 2007). Tal contexto revela a importância do desenvolvimento de práticas pedagógicas que viabilizem melhores resultados na aprendizagem dessa disciplina. Assim, o objetivo desta pesquisa foi analisar a contribuição do uso de softwares para o ensino de lógica, adotando-se o Dojo como prática pedagógica (ROOKSBY; HUNT; WANG, 2014; 
ROCHA, Fabio Gomes; NASCIMENTO, Ester Fraga Vilas-Bôas Carvalho do. O uso do Dojo na prática pedagógica do ensino de lógica de programação.

BOSSAVIT; GAILLOT, 2005; BRAVO; GOLDMAN, 2010; LUZ; SECA NETO; NORONHA, 2013; SATO; BRAVO; CORBUCCI, 2008). Trata-se de uma técnica de treinamento para programadores, baseada em testes, idealizada por David Thomas, que iniciou essa prática em 2003, em Paris, chegando no Brasil em 2007, com Ivan Sanchez, a partir da criação do grupo Dojo Floripa (BACHE, 2013). O Dojo constitui-se em um "[...] espaço onde programadores se reúnem para treinar e aprender. As reuniões são periódicas e centradas num desafio de programação" (CODING DOJO, 2013, tradução nossa). A prática do Dojo, segundo Estácio (2017) é desenvolvida em grupo, o qual deve trabalhar em conjunto para solucionar determinado problema. Dessa forma, a prática promove um ambiente de aprendizagem colaborativa e contínua.

Embora se identifique, ainda, pouca literatura sobre a aplicação do Conding Dojo na aprendizagem, conforme constatado nos recentes estudos de Estácio (2017), alguns resultados se mostram promissores. Como exemplo tem-se a pesquisa de Sato, Bravo e Corbucci (2008), que exploram a aplicação do Dojo relatando como estudantes com diferentes níveis de habilidade compartilham seu conhecimento em um grupo de trabalho amplo. Outras investigações, como as de Heinoven et al. (2013), Luz, Seca Neto e Noronha (2013) apontam, que a prática de Conding Dojo permite que os alunos participem ativamente do processo de aprendizagem por meio do comportamento colaborativo. Em adição a esses estudos, a pesquisa de Li, Plaue e Kraemer (2013) indicou que, quando adotada a programação em par, há redução no número de evasão nas disciplinas iniciais de cursos superiores de Computação. No entanto, na experiência de Salleh, Mendes e Grundy (2011), observa-se que esse modelo de trabalho resulta em melhor nível de satisfação por parte dos alunos quando o grupo apresenta habilidades e motivações semelhantes.

Nesse contexto, considerando a indicação de Dewey (1965, p. 16) de que a “[...] experiência educativa, é pois, essa experiência inteligente, em que participa o pensamento, através do qual se vêm a perceber relações e continuidades antes não percebidas", entendeu-se possível a verificação da hipótese de que a aplicação pedagógica do Dojo poderia viabilizar melhor assimilação por parte dos alunos, reduzir o índice de reprovação e de evasão, bem como ampliar a interação entre os alunos e entre eles e o professor, além de expandir a visão abstrata para solução de problemas. 
ROCHA, Fabio Gomes; NASCIMENTO, Ester Fraga Vilas-Bôas Carvalho do. O uso do Dojo na prática pedagógica do ensino de lógica de programação.

\section{Metodologia}

Como investigação empírica, a pesquisa constitui um estudo de caso, uma vez que se voltou à observação de um grupo específico de alunos (YIN, 2009), tornando-se exploratória, sobre a verificação das dificuldades de aprendizagem dos estudantes, e descritiva, quanto à experimentação do uso do Dojo como metodologia para o ensino. As análises sobre os resultados foram feitas sob abordagem qualitativa, associando-se os dados obtidos na atividade pedagógica e os referenciais adotados no estudo. O público investigado constituiu-se de alunos da disciplina de Lógica de Programação, a qual possui 140 horas do total de 1.100 horas do curso Técnico de Informática do Serviço Nacional de Aprendizagem Industrial do Estado de Sergipe, no período de março a abril de 2014.

Esse curso, com ingresso anual e denominado Ensino Articulado, é desenvolvido pelo Serviço Social da Indústria (SESI) e Serviço Nacional de Aprendizagem Industrial (SENAI), onde os alunos frequentam o ensino médio no SESI, concomitantemente ao ensino técnico na segunda instituição. As turmas investigadas, com idade média dos alunos entre 14 e 16 anos, estavam distribuídas entre os turnos da manhã e tarde, com estudantes de ambos os sexos, conforme exposto no Quadro 1, a seguir:

Quadro 1 - Distribuição das turmas e sexo dos alunos

\begin{tabular}{|c|c|c|c|}
\hline Turno & Homens & Mulheres & Total \\
\hline Manhã & 20 & 05 & 25 \\
\hline Tarde & 19 & 10 & 29 \\
\hline Total & 39 & 15 & 54 \\
\hline
\end{tabular}

Fonte: Elaborado pelo autor.

Iniciou-se o ensino da disciplina com oito horas de abordagem teórica sobre Lógica Aplicada à Programação, contextualizando-a diante da formação do Técnico em Informática e identificando a relevância para o desenvolvimento das atividades pertinentes a esse profissional. Em prosseguimento ao plano de ensino, explorou-se a relação da lógica com situações vivenciadas no cotidiano dos estudantes, buscando-se os conhecimentos implícitos por meio de exemplos, como o fato cotidiano da tomada de decisão no momento em que um indivíduo 
ROCHA, Fabio Gomes; NASCIMENTO, Ester Fraga Vilas-Bôas Carvalho do. O uso do Dojo na prática pedagógica do ensino de lógica de programação.

atravessa uma rua. Essa ação demanda a avaliação sobre um semáforo estar sinalizando o impedimento ou liberação para o pedestre.

A partir dessas aulas introdutórias, passou-se a adotar a ferramenta Scratch como incentivo à atuação das turmas em grupos de quatro estudantes para a solução de problemas. $\mathrm{O}$ Scratch é um software, criado pelo Massachusettes Institute of Technology (MIT) para o ensino de lógica. Ele permite que o aluno interaja de forma simples, e crie animações virtuais com base na aplicação de lógica.

Após três aulas, totalizando 12 horas, prosseguiu-se com a apresentação do conceito, dos objetivos e das regras para o uso do Dojo na busca de soluções de problemas. Todos os alunos foram envolvidos na solução de um único problema no software VisualG. Nessa dinâmica, foi proposto um desafio. O objetivo da atividade não se relaciona à conclusão do desafio, mas ao aprender com as experiências vivenciadas pelo grupo. Aplicando-se os princípios de Dewey (1965), no tocante à atribuição de uma tarefa ao estudante mantendo-o sob orientação nas etapas de execução, uma dupla iniciou o trabalho de codificação de um computador, sendo a atividade exposta ao grande grupo para que todos pudessem acompanhar. Um dos estudantes do grupo assumiu o papel de "piloto", desenvolvendo a codificação, enquanto um segundo membro do grupo atuou como "co-piloto", observando e auxiliando o piloto.

Cada grupo recebeu o tempo de cinco a sete minutos para desenvolver a atividade. $\mathrm{Na}$ conclusão desse tempo, o piloto devia juntar-se ao grande grupo, passando o co-piloto ao lugar do primeiro. Isso se estendeu a todos do grupo, até a solução do problema proposto. Ao finalizar, iniciou-se o ciclo de prática de codificação, visando a melhoria do código desenvolvido inicialmente. A intenção pedagógica era despertar no estudante o entendimento de que uma solução inicial pode ser otimizada por outras contribuições, não se tornando a única possível, promovendo, assim, um ambiente de integração social entre os estudantes (DEWEY, 1965). As aulas seguintes foram intercaladas com conteúdos teóricos, exercícios individuais e novas proposições de soluções com o uso do Dojo, induzindo à prática dos conteúdos trabalhados nas aulas teóricas., cada quatro horas de matéria teórica e exercícios foram seguidas de quatro horas de uso do DOJO, promovendo um ciclo de aprendizado inerentemente social por meio da integração dos alunos (DEWEY, 1965). 
ROCHA, Fabio Gomes; NASCIMENTO, Ester Fraga Vilas-Bôas Carvalho do. O uso do Dojo na prática pedagógica do ensino de lógica de programação.

\section{A abordagem conceitual sobre a prática pedagógica utilizada}

A utilização do software Scratch para a experiência pedagógica tomou como base a teoria construcionista, a qual "[...] atribui especial importância ao papel das construções no mundo como apoio para o que ocorreu na cabeça, tornando-se, deste modo menos uma doutrina puramente mentalista" (PAPERT, 1980, p. 142). Essa ferramenta tecnológica tem uma interface simples, que permite ao aluno interagir com personagens virtuais, possibilitando, ainda, a criação de estórias interativas, jogos e animações. O seu foco é auxiliar os jovens a pensar de forma criativa, sistemática e colaborativa (SCRATCH, 2013). O público alvo do software são jovens entre 8 a 16 anos, estando, assim, a sua aplicação ao curso investigado dentro do escopo de idade previsto.

As atividades pedagógicas propostas foram estabelecidas com base nos estágios de Piaget (1968), considerando o estágio operacional formal, ocorrido a partir dos 11 ou 12 anos até a vida adulta do indivíduo. Aplicando-se os pressupostos desse estágio de desenvolvimento humano, na medida em que o aluno decompõe o problema em etapas, partindo dos problemas gerais, em uma visão concreta, aos complexos, desenvolvendo uma visão mais abstrata, se estabelece o pensamento dedutivo-indutivo necessário à lógica.

Ao posicionar os alunos como participantes ativos do processo de solução de problemas, também se posiciona eles diante do concreto, das suas experiências mais próximas, em um processo de interação pessoa-meio conforme descrito por Bigge (1982) sobre a realidade percebida. O professor e os colegas ficam, na dinâmica proposta, também envolvidos nessa interação com vistas ao conhecimento, já que esse movimento "[...] implica uma relação sujeitosujeito-objeto" (GAMEZ, 2013, p. 83). Sendo a sala de aula o local em que ocorreu a atividade, esse espaço "[...] pode assumir para si a perspectiva de interação com o conhecimento e com os atores do ato educativo, Assume também a função de ser o principal lugar em que se desenvolva a inteligência coletiva" (KENSKI, 2007, p. 47).

$\mathrm{O}$ ato de codificar um software resulta na criação de um objeto com significância ao aluno, já que vê a concretização da aplicação de seus conhecimentos. Para tal criação o aluno é estimulado a perceber o problema de forma individual, mas sendo levado a considerar a participação colaborativa dos demais na elaboração da solução lógica. Desta forma, os alunos 
ROCHA, Fabio Gomes; NASCIMENTO, Ester Fraga Vilas-Bôas Carvalho do. O uso do Dojo na prática pedagógica do ensino de lógica de programação.

passam a ter uma participação ativa e interativa, a qual é mais positiva que a recepção passiva e é incentivada por meio da prontidão e da aprendizagem (BIGGE, 1982).

$\mathrm{Na}$ prática investigada, a participação do professor é especialmente relevante nos momentos da passagem da abordagem teórica para a explicação detalhada sobre a forma da busca de solução ao problema proposto. Além disso, é fundamental que o professor esclareça sobre a possibilidade da solução proposta ser revista pelos alunos, constituindo um ciclo contínuo de melhoria da solução inicial. O processo de ensino e da aprendizagem torna-se, assim, contínuo na própria reconstrução da experiência (DEWEY, 1997).

No entanto, a distribuição desses momentos de prática ao longo da disciplina investigada não deve levar os alunos à exaustão, devendo ser evitada a realização diária, pois conforme comprovam as pesquisas, a prática espaçada é mais eficiente que a prática compacta (BIGGE, 1982).

\section{Considerações finais}

A lógica, por ser essencial ao ensino que envolva programação, exige do aluno a compreensão de conceitos e aplicações que perpassarão toda a sua formação e o acompanharão em sua jornada profissional. Ao professor dessa disciplina compete criar um ambiente motivador e atraente, desenvolvendo a cognição dos alunos. A experiência tratada nesta pesquisa, com a utilização do Dojo como instrumento pedagógico, demonstrou-se positiva, com resultados relevantes. Entre eles, constou o índice zero de evasão, corroborando as observações de Li, Plaue e Kraemer (2013) sobre a redução na desistência de alunos, e o índice de 6\% de faltas, o qual demonstra maior interesse de participação dos alunos, conforme encontrado também nos estudos de Luz, Seca Neto e Noronha (2013), e Heinonen et al. (2013). Em turmas anteriores, as quais tiveram o mesmo professor e práticas pedagógicas sem o uso do DOJO, as faltas às aulas atingiram 23\%. Ao final da dinâmica na experiência aqui relatada, os alunos alegaram estar motivados, e, por isso, evitavam faltar às aulas.

Embora a experiência tenha a limitação de aplicação a duas turmas, é possível observar que o conjunto interação e aplicação criativa viabiliza uma melhor assimilação por parte dos alunos. Nas avaliações aplicadas em período anterior ao uso do Dojo constaram médias entre a nota seis e sete, sendo seis a mínima para aprovação. Após a experiência, as notas elevaram-se 
ROCHA, Fabio Gomes; NASCIMENTO, Ester Fraga Vilas-Bôas Carvalho do. O uso do Dojo na prática pedagógica do ensino de lógica de programação.

para a média entre sete vírgula cinco e oito, ressaltando-se a opinião dos alunos sobre a satisfação obtida na atividade proposta.

Considerando que a disciplina subsequente é a de Programação Desktop, os alunos se apresentam melhor preparados nos processos lógicos necessários também aos demais conhecimentos a serem trabalhados no curso. Dessa forma, mesmo que se considere a relevância do aprofundamento da experiência por meio de um maior período de observação e da aplicação em outras turmas, os resultados obtidos já permitem constatar a viabilidade de adoção do Dojo como prática pedagógica para o desenvolvimento do pensamento lógico, abstrato e sistêmico.

Conclui-se, assim, que o papel das atividades e ferramentas utilizadas é o de auxiliar o professor no processo de ensino e da aprendizagem, tornando-o mais atrativo, interativo e colaborativo. Ponderando-se que a pesquisa não pretende esgotar as possibilidades de sucesso com outras práticas, novos estudos podem ser desenvolvidos para a busca de variadas ferramentas a serem aplicadas ao ensino da lógica, como jogos e uso de robótica lego.

\section{Referências}

BACHE, Emily. The coding DOJO handbook: a practical gruide to creatins a spacce where good programmers can become great programmers. Leanpub: Vancouver, 2013.

BIGGE, Morris L. Learning theories for teachers. New York: Harper \& Row Publisher, 1982.

BOSSAVIT, L.; GAILLOT, E. The Coder's Dojo: a different way to teach and learn Programming. In: INTERNACIONAL CONFERENCE ON AGILE SOFTWARE DEVELOPMENT XP, 6., 2005, Proceedings... Sheffield, 2005. p. 290-291.

BRASIL. Decreto n 70.370 , de 5 de abril de 1972. Cria a Comissão de Coordenação das Atividades de Processamento Eletrônico, e dá outras providências. Diário Oficial [da] República Federativa do Brasil, Brasília, DF, 6 abr. 1972a. Seção 1, p. 2990.

BRASIL. Conselho Federal de Educação. Parecer n ${ }^{\circ} .45$, de 12 de janeiro de 1972. A qualificação para o trabalho no ensino de $2^{\circ}$ grau. O mínimo a ser exigido em cada habilitação profissional. Documenta, Brasília, n. 134, p. 107-155, jan, 1972 b.

BRAVO, Mariana, GOLDMAN, Alfredo. Reinforcing the learning of agile practices using coding Dojos. In: INTERNACIONAL CONFERENCE ON AGILE SOFTWARE DEVELOPMENT XP, 11, 2010, Proceedings... Trondheim, 2010. p. 379-380.

CABRAL, Maria Izabel Cavalvanti et al. A trajetória dos cursos de graduação da área de computação e informática: 1969-2006. Rio de Janeiro: SBC, 2008.

CODING DOJO. DOJO. Disponível em: <https://codingdojo.org/>. Acesso em: 12 nov. 2013.

DEWEY, John. Vida e educação. São Paulo: Melhoramentos, 1965.

DEWEY, John. Experience and education. New York: Touchstone, 1997. 
ROCHA, Fabio Gomes; NASCIMENTO, Ester Fraga Vilas-Bôas Carvalho do. O uso do Dojo na prática pedagógica do ensino de lógica de programação.

ESTÁCIO, Bernardo José da Silva. Uma avaliação empírica sobre a aprendizagem colaborativa em conding dojo randori no contexto de desenvolvimento de software. 2017, 173f. Tese (Doutorado em Ciências da Computação) - Programa de Pós-Graduação em Ciências da Computação, Pontifícia Universidade Católica do Rio Grande do Sul, Porto Alegre, 2017.

GAMEZ, Luciano. Psicologia da educação. Rio de Janeiro: LTC, 2013.

HEINONEN, Kenny et al. Learning agile software engineering practices using coding dojo. In: THE ANNUAL ACM SIGITE CONFERENCE ON INFORMATION TECHNOLOGY EDUCATION SIGITE. 13., 2013. Proceedings... Orlando, 2013. p. 97-102.

INSTITUTO FEDERAL DE SÃO PAULO. Plano de Desenvolvimento Institucional 2014-2018.

Disponível em: <http://www.ifsp.edu.br/index.php/documentos-institucionais/pdi.html>. Acesso em: 25 maio 2017.

KENSKI, Vania Moreira. Educação e tecnologias: o novo ativo da informação. Campinas: Papirus, 2007.

LI, Zen; PLAUE, Christopher; KRAEMER, Eileen. A spirit of camaraderie: the impact of pair programming on retention. In: SOFTWARE ENGINEERING EDUCATION CONFERENCE. 2013. Proceedings... San Francisco, 2013. p. 209-218.

LUZ, R. B.; SECA NETO, A. G. S.; NORONHA, R. V. Teaching TDD: the coding Dojo style. In: INTERNATIONAL CONFERENCE ON ADVANCED LEARNING TECHONOLOGIE. 2013. Proceedings... Beijing, 2013. p. 371-375.

MANZANO, José Augusto N. G.; OLIVEIRA, Jayr Figueiredo de. Algoritmos: lógica para desenvolvimento de programação. São Paulo: Érica, 2009.

PAPERT, Seymour. Mindstorms: children, computers, and powerful ideas. New York: Basic Books, 1980.

PIAGET, Jean. Six psychological studies. New York: Vintage Books, 1968.

PIMENTEL, E. P. et al. De computadores. In: WORKSHOP SOBRE EDUCAÇÃO EM COMPUTAÇÃO - SBC. 2003. Proceedings... Porto Alegre, 2003. p. 105-116.

RICE, J. R.; ROSEN, S. The Origins of Computing at Purdue University. In: RICE, John; DEMILLO, Richard A. Studies in computer science: in honor of Samuel D. Conte. New York: Springer Science, 1994. p. 31-44.

ROOKSBY, J.; HUNT, J.; WANG, X. The theory and practice of Randori Coding Dojos. In: INTERNATIONAL CONFERENCE ON AGILE SOFTWARE DEVELOPMENT XP, 15., 2014. Proceedings... Roma, 2014. p. 251-259.

SALLEH, Norsaremah; MENDES, Emilia; GRUNDY, John C. Empirical studies of pair programming for CS/SE teaching in higher education: A systematic literature review. IEEE Transactions on Software Engineering, Estados Unidos, v. 37, n. 4, p. 509-525, 2011.

SATO, D.; BRAVO, M.; CORBUCCI, H. Coding Dojo: an environment for learning and sharing Agile practices. In: AGILE CONFERENCE - IEEE. 2008. Proceedings... Toronto, 2008. p. 459-464.

SEBESTA, Robert. W. Concepts of programming language. 10. ed. Harlow: Pearson, 2013.

SCRATCH (SOFTWARE). Scratch. Massachusettes Institute of Technology (MIT). Disponível em: <http://scratch.mit.edu>. Acesso em: 12 nov. 2013.

SILVEIRA, Zuleide Simas da. Contradições entre capital e trabalho: concepções de educação tecnológica na reforma do ensino médio e técnico. 2007. 311f. Dissertação (Mestrado em Educação) Universidade Federal Fluminense, Niterói, 2007. 
ROCHA, Fabio Gomes; NASCIMENTO, Ester Fraga Vilas-Bôas Carvalho do. O uso do Dojo na prática pedagógica do ensino de lógica de programação.

WORLDSKILLS. WorldSkills International History. Santo André: Ipsis Gráfica, 2010.

WORLDSKILLS. Site oficial de fotografias da WorldSkills. Disponível em: <https://www.flickr.com/photos/worldskills/>. Acesso em: 25 maio 2017.

YIN, Robert K. Case study research: design and methods. Califórnia: Sage, 2009.

Fabio Gomes Rocha

Universidade Tiradentes | Núcleo de Computação

Aracaju | SE | Brasil. Contato: gomesrocha@gmail.com

ORCID 0000-0002-0512-5406

Ester Fraga Vilas-Bôas Carvalho do Nascimento

Universidade Tiradentes | Programa de Pós-Graduação em

Educação

Aracaju | SE | Brasil. Contato: esterfraga@gmail.com

ORCID 0000-0002-4050-767X

Artigo recebido em: 6 nov. 2017 e aprovado em: 7 dez. 2017. 